\title{
Étude ethnobotanique des plantes médicinales utilisées dans le traitement des affections dermatologiques dans le plateau central marocain
}

\author{
Fatima El hilah, Fatiha Ben akka, Rachid Bengueddour, Atmane Rochdi, Lahcen Zidane. \\ Laboratoire de Biodiversité et Ressources Naturelles, Université Ibn Tofaïl, Faculté des Sciences, BP 133, Kénitra, \\ Maroc. \\ Auteur de la correspondance : elhilah@gmail.com.
}

Original submitted in on 24th November 2015. Published online at www.m.elewa.org on $29^{\text {th }}$ February 2016 http://dx.doi.org/10.4314/jab.v98i1.2

\section{RÉSUMÉ}

Objectif : Une étude ethnobotanique réalisée au niveau du plateau central marocain durant 2010 à 2013, pour objectif de décrire les plantes médicinales utilisées dans le traitement des affections dermatologiques. Cette étude est une évaluation scientifique du potentiel médicinal des plantes, pour une meilleure valorisation de ces ressources naturelles et de ce savoir faire traditionnel.

Méthodologie et résultats : Lors de chaque entretien, à l'aide d'un questionnaire, nous avons collecté toute l'information sur l'enquêté et les plantes médicinales utilisées par celui-ci. L'enquête a ciblé 737 personnes de la population locale, dont 559 personnes utilisent les plantes médicinales $(76,40 \%)$ et 178 personnes ont recours à la médecine moderne $(23,60 \%)$. Dans cette étude, nous nous sommes intéressés à la première catégorie qui utilise les plantes médicinales. Elle comprend 365 femmes $(65,60 \%)$ et 194 hommes $(34,40 \%)$, âgées de 10 à 90 ans. $67 \%$ des usagers des plantes médicinales sont des analphabètes.

Ainsi, l'enquête a permis de recenser 51 espèces de plantes regroupées en 47 genres et appartenant à 32 familles dont les Lamiacées, qui sont majoritaires, suivis des Myrtacées, des Cactacées et des Lythracées.

Les feuilles sont utilisées dans le traitement des infections dermatologiques. Les remèdes sont préparés essentiellement en poudre.

Conclusion et application des résultats : Cette étude fait ressortir les plantes médicinales utilisées dans le traitement des affections dermatologiques par la population du plateau central marocain. Les résultats obtenus constituent une source d'informations très précieuse pour la région étudiée et pour la flore médicinale nationale. Ils pourraient être une base de données pour les recherches ultérieures dans les domaines de la phytochimie et de la pharmacologie et dans le but de chercher de nouvelles substances naturelles.

Mots clés : plantes aromatiques et médicinales, ethnobotanique, médecine traditionnelle, affections dermatologiques, plateau central, Maroc.

\section{ABSTRACT}

Objective: An ethnobotany study in Moroccan central plateau between 2010 and 2013 was set with the objective of evaluating the potential of the region on medicinal plants used in the treatment of Dermatological diseases. This study is a scientific evaluation of the potential medicinal plants, for better utilization of natural resources and the traditional knowledge. 
Methodology and results: During each interview, all the information on the survey and the medicinal plant used was collected. The survey targeted 737 people from the local population with 559 were using medicinal plants $(76.40 \%)$ and 178 people using modern medicine $(23.60 \%)$. The population that cared for medicinal plants included 365 women $(65.60 \%)$ and 194 men $(34.40 \%)$, aged 10 to 90 years and all users of traditional medicine were illiterate and dominated with a percentage of $84.21 \%$. Thus, the investigation identified 51 plant species grouped in 47 genera and 32 families belonging to the Lamiaceae, which were the majority, followed by the Myrtaceae, the Cactaceae and the Lythraceae. The leaves were used in the treatment of dermatological diseases. The remedies were prepared primarily in powder.

Conclusion and application of results: This study shows the medicinal plants used in the treatment of dermatological complaints by the population of the Moroccan central plateau. These results constitute a source of very precious information for the area studied and for subsequent researches for the domains of the phytochemistry and pharmacology in order to search for new natural substance.

Key words: Aromatic and medicinal plants, ethnobotanical, traditional medicine, dermatological diseases, central plateau, Morocco.

\section{INTRODUCTION}

Depuis plusieurs années, l'homme qui vit côte à côte avec les plantes, est habitué à les consommer pour leurs propriétés médicinales et nutritives. Les produits naturels présentent un grand intérêt comme matière première destinée aux différents secteurs d'activité tels que: le cosmétique, la pharmacie, l'agroalimentaire, le phytosanitaire et l'industrie (Selles C., 2012). Ainsi, l'utilisation des remèdes à base de plantes connaît dernièrement un engouement sans précédent. De plus en plus de gens sont à la recherche de médicaments "naturels" et il semblerait même que les cosmétiques et les produits d'entretien à base de plantes soient aujourd'hui de plus en plus utilisés (Adossides A., 2003). Par ailleurs, plusieurs remèdes sont utilisés tous les jours par de nombreuses populations pour les soins de santé. De même pour la population du plateau central marocain, vu la diversité des plantes

\section{MATERIEL ET METHODES}

A-Zone d'étude : Les enquêtes ont été menées dans la Meseta centrale, souvent appelée massif central marocain, qui est limité au nord par le couloir sud-rifain (Maamora et plateau de Meknès), au sud par le plateau des phosphates et par le Moyen Atlas à l'Ouest (Figure 1). La Meseta centrale constitue un haut pays dont l'altitude moyenne dépasse $500 \mathrm{~m}$ (Combe et al., 1975). Le massif central marocain est constitué de granites et de roches métamorphiques qui affleurent essentiellement en trois massif distincts : au sud de Rommani, au nord-ouest d'Aguelmous et à l'Ouest d'Oulmes. Les schistes primaires (Ordovicien, Silurien, Dévonien ou Carbonifère) médicinales ayant un grand pouvoir thérapeutique, elle adopte la médication par les plantes pour des pathologies diverses; et dans cette gamme, nous avons noté une place importante occupée par les affections dermatologiques. Les maladies dermatologiques sont nombreuses et très répandues, elles sont considérées comme un ensemble de pathologies dont les symptômes les plus visibles se manifestent au niveau des organes cibles notamment la peau, les muqueuses et les phanères. Les études ethnobotaniques que nous menons depuis 2010 auprès de la population du plateau central visent à une meilleure connaissance de la diversité des plantes médicinales, les remèdes utilisés contre les affections dermatologiques et valoriser se savoir faire en vue d'une exploitation durable des ressources renouvelables de la biodiversité végétale.

sont très largement présents dans la Meseta, où ils sont fréquemment injectés de filons dolomitiques souvent minéralisés. En intercalation dans les schistes se rencontrent des bancs de quartzites ou de grés dont la puissance varie de quelques mètres à quelques dizaines de mètres. Enfin, quelques calcaires d'âge essentiellement Dévonien apparaissent dans des secteurs localisés et ne représentent que des volumes peu importants (Beaudet, 1969). Le climat varie avec la continentalité et l'altitude. II est semi-aride en basse altitude et subhumide en haute altitude. La complexité topographique de la région, l'interférence de l'altitude et 
de la continentalité sur la bioclimatologie influente sur la répartition de la végétation. On trouve ainsi des zones forestières, les versants des grandes vallées sont presque exclusivement le domaine du chêne-liège sur des sols silicieux, et de l'oléastre, du lentisque et de Tizra sur sols calcaires ou argileux. Aussi, le chêne-vert s'y introduit souvent vers le Sud-Est, mais disparaît vers le Sud. Les crêtes portent une flore riche en espèces endémiques et le jujubier devient très abondant en approchant du plateau des phosphates (Combe et al., 1975). Vu que les terres cultivables sont rares, le pastoralisme est l'activité prédominante. Par conséquent, la population est en grande partie non sédentarisée et seules quelques agglomérations s'organisent autour des marchés locaux (Combe et al., 1975).

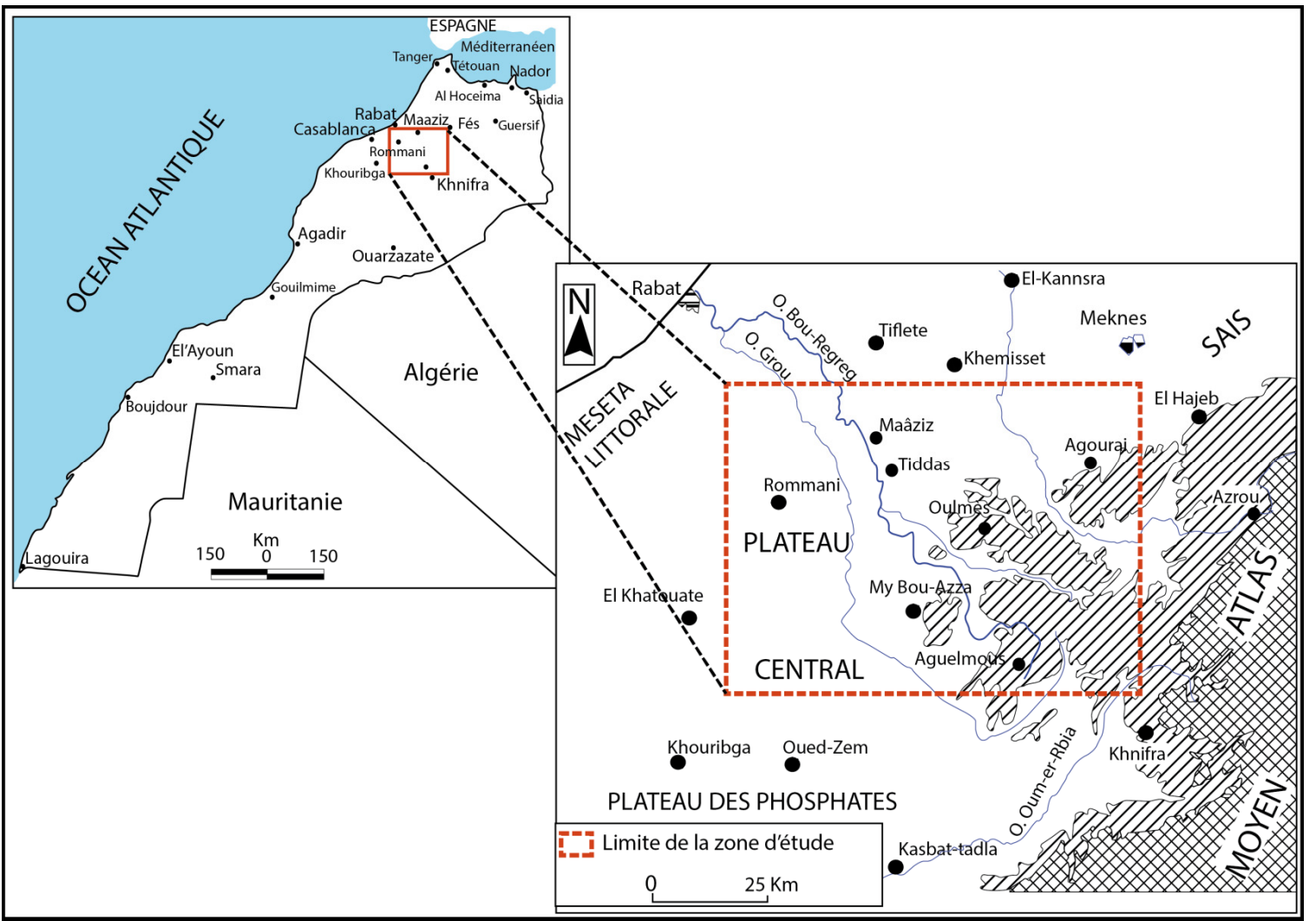

Figure 1 : Situation géographique de la zone d'étude (Combe et al., 1975).

B- Échantillonnage : $\mathrm{Au}$ cours de cette étude ethnobotanique des plantes médicinales, nous avons choisi 43 stations. La localisation des différents points d'enquêtes est repérée par la méthode d'échantillonnage stratifié. Pour effectuer ce type d'échantillonnage, on s'est basé sur trois facteurs ; la végétation (chêne liège, chêne vert, chêne zéene, formation secondaires), le climat (subhumide ou semi aride), et l'altitude.

C- Questionnaire : La méthode d'étude est basée sur une fiche questionnaire ethnobotanique soumise aux enquêtés au cours d'entretiens individuels. La fiche d'enquête ethnobotanique a été remplie auprès de 737 personnes. Ce questionnaire concerne le profil de chaque enquêté et les données ethnopharmacologiques recueillies pour chaque plante utilisée contre les affections dermatologiques.

D- Collecte des données : L'enquête ethnobotanique a durée une période allant de 2010 à 2013. Pour collecter les données ethnobotaniques, un échantillon de 737 personnes a été interrogé aléatoirement sur 43 points relevés comprenant des villes, des villages, des douars et des souks. Les enquêtés ont été questionnés individuellement à l'aide d'une fiche questionnaire remplie par interrogation orale. Certaines données collectées lors des enquêtes sont relatives à l'informateur (âge, sexe, niveau d'étude, situation familiale, habitat), et d'autres portent sur les plantes médicinales telles que le nom local de l'espèce, les organes utilisés, leurs modes de préparation et d'administration. 
E- Identification des espèces : Certaines espèces inventoriées sont identifiées sur le terrain. D'autres au Laboratoire de Biodiversité et Ressources Naturelles, de la Faculté des Sciences de Kénitra, grâce à la flore pratique du Maroc et la nouvelle flore de l'Algérie et des Régions désertiques méridionales: Fennane $M$., Ibn Tattou M., Mathez J., Ouyahya A., Eloualidi J., 1999. Flore pratique du Maroc, manuel de détermination des plantes vasculaire. Volume 1: ptéridophyte gymnospermes, angiosperme (Lauraceae-Neuradaceae). Travaux de l'institut scientifique, série botanique $n^{\circ} 36$. Rabat. 558p. Fennane M., Ibn Tattou M., Ouyahya A., Eloualidi J., 2007. Flore pratique du Maroc, manuel de détermination des plantes vasculaire. Volume 2 : angiosperme (Leguminoseae- Lentibulariaceae). Travaux de l'institut scientifique, série botanique $n^{\circ} 38$. Rabat.

\section{RESULTATS ET DISCUSSION}

Choix entre la médecine traditionnelle et la médecine moderne: L'enquête ethnobotanique réalisée sur le terrain a permis d'interroger 737 personnes comprenant 636p. Fennane. M., Ibn Tattou. M., Eloualidi. J., 2014. Flore pratique du Maroc, manuel de détermination des plantes vasculaires. Volume 3: Dicotylédones, Monocotylédones. Travaux de l'institut scientifique. Université Mohamed V. Rabat. 793p. Quezel P., Santa S., 1962a. Nouvelle flore de l'Algérie et des Régions désertiques méridionales, (Tome I). Edition centre national de la recherche scientifique. $565 p$.

Quezel P., Santa S., 1962b. Nouvelle flore de l'Algérie et des Régions désertiques méridionales, (Tome II). Edition centre national de la recherche scientifique. p571-p1165.

F- Traitement informatique : Les données recueillies et notées sur les fiches d'enquête ont été ensuite saisies et analysées statistiquement par le logiciel SPSS (System Package for Social Sciences, version 11,5) et Excel 2007.

des femmes et des hommes, dont 563 ont recours à la médecine traditionnelle $(76,40 \%)$ et 174 à la médecine moderne $(23,60 \%)$.

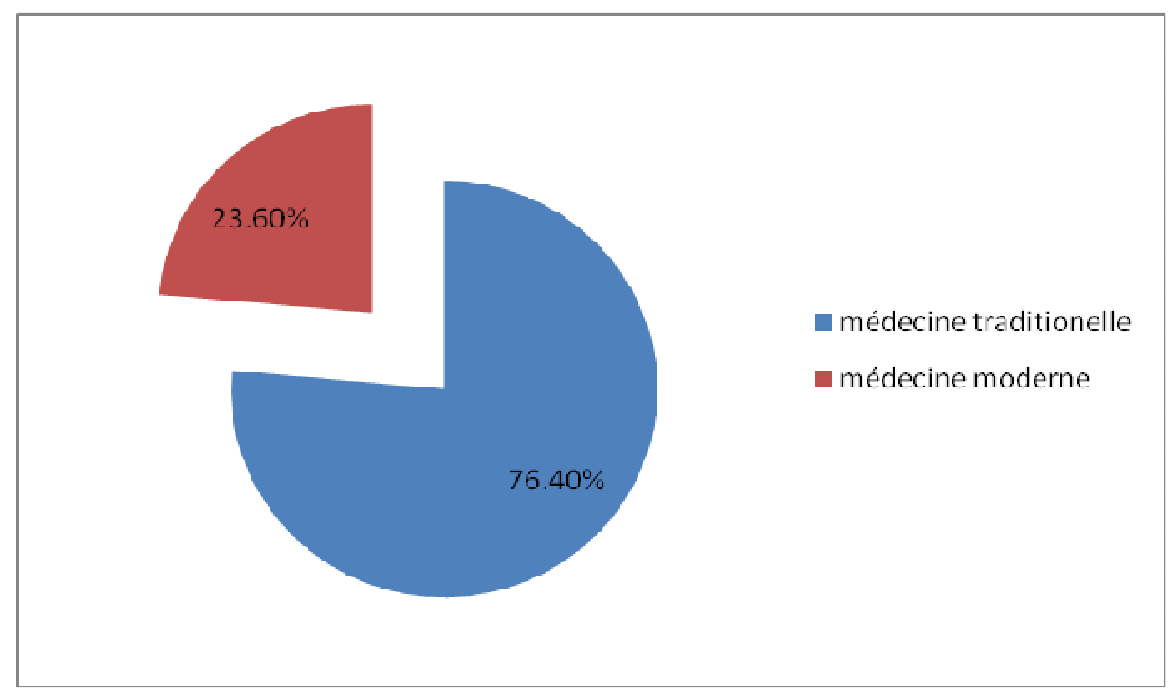

Figure 2 : Le pourcentage du choix entre la médecine traditionnelle et la médecine moderne

\section{Utilisation des plantes médicinales selon le profil des enquêtés}

Selon le genre : Dans cette région, les usagers qui ont recours à la médecine traditionnelle sont de l'ordre de 563 personnes. Par ailleurs, les hommes ainsi que les femmes sont concernés par la médecine traditionnelle. Cependant, les femmes utilisent plus les plantes médicinales. Ainsi, 64,63\% des utilisateurs des plantes médicinales sont des femmes, contre $35,37 \%$ des hommes.
Selon l'âge : Les personnes d'âge entre [40-49] ans ont une fréquence d'utilisation des plantes médicinales de $34,45 \%$. Viennent ensuite les tranches d'âge [30-39], [50-59], [60-69], [20-29] avec respectivement 18\%, 17,07 $\%, 13,72 \%, 4,57 \%$. Les personnes d'âge supérieur de 70 ans ont $10,97 \%$ et enfin celle de moins de 19 ans ont $1,22 \%$.

Selon le niveau d'étude : Le traitement des données nous a permis d'obtenir le graphique de la figure 4, qui montre qu'au plateau central $78,66 \%$ des personnes utilisatrices des plantes médicinales sont des 
analphabètes, viennent ensuite les niveaux primaire, secondaire et universitaire avec un pourcentage respectivement de $12,20 \%, 5,80 \%, 3,34 \%$.

Selon la situation familiale : L'examen des données montrent que les plantes médicinales sont beaucoup plus

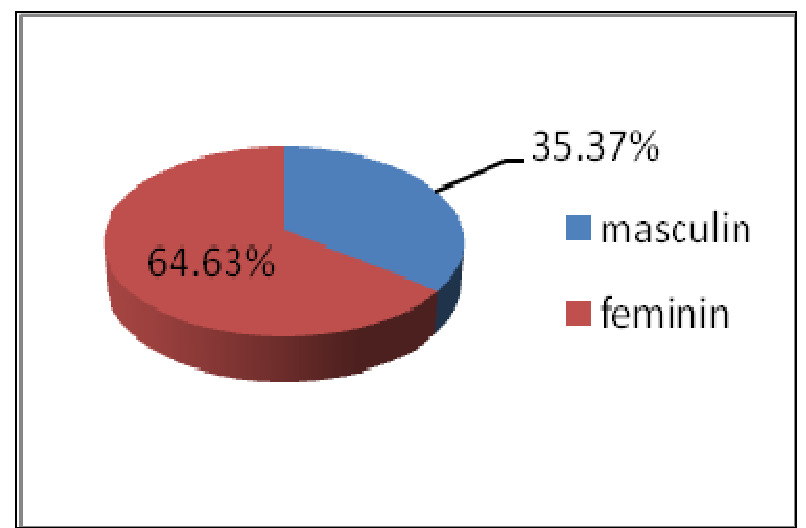

Figure 3 : Répartition de la fréquence d'utilisation des plantes médicinales selon le genre dans le Plateau central.

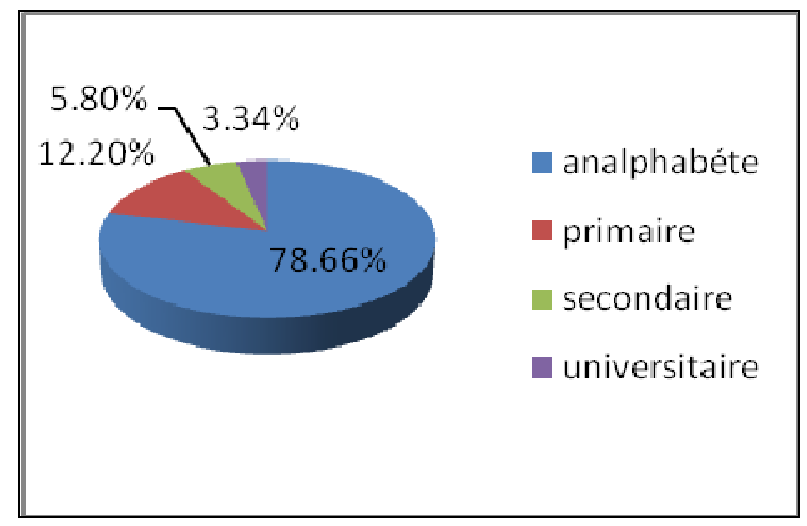

Figure 5 : Répartition de la fréquence d'utilisation des plantes médicinales selon le niveau d'étude dans le Plateau central.

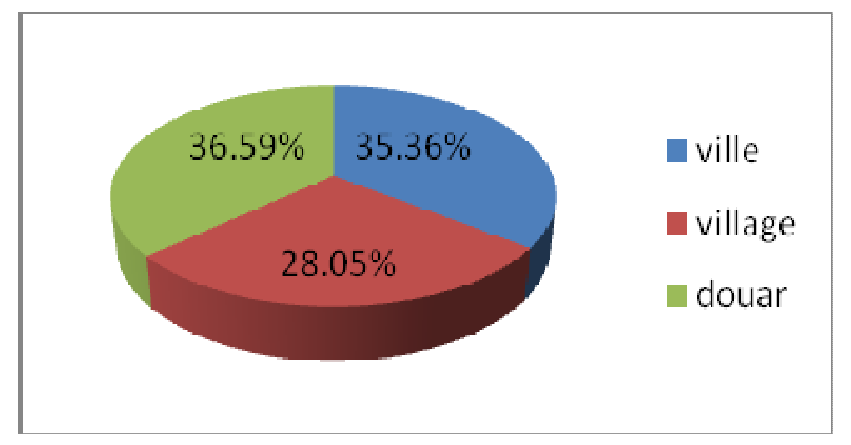

utilisées par les personnes mariées $(88,10 \%)$, par contre les célibataires ne représentent que $(11,90 \%)$ (Figure 5).

Selon l'habitat : L'utilisation des plantes médicinales est répandue sur tout le plateau central. En effet, la population étudiée est répartie entre les douars $(36,59 \%)$, les villes $(35,36 \%)$, et les villages $(28,05 \%)$.

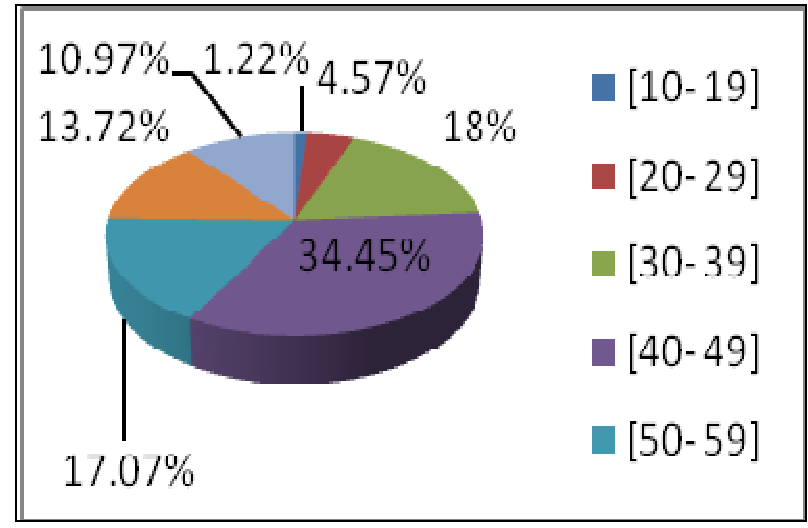

Figure 4 : Répartition de la fréquence d'utilisation des plantes médicinales par classe d'âge dans le Plateau central.

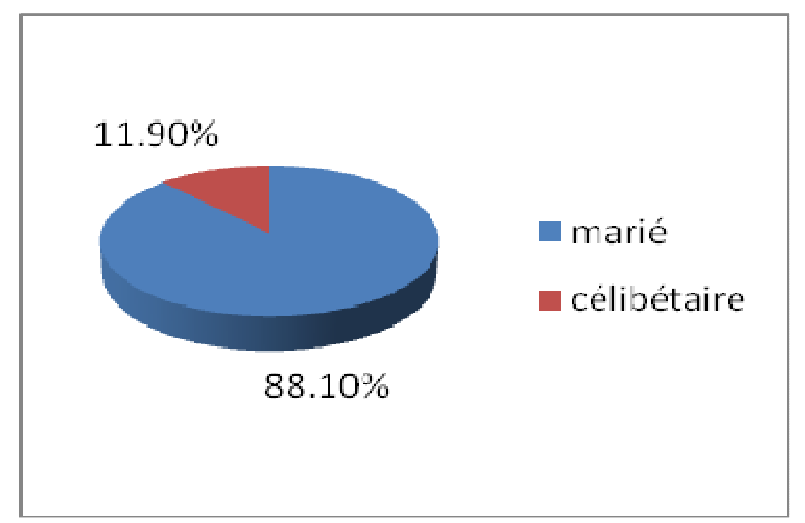

Figure 6 : Répartition de la fréquence d'utilisation des plantes médicinales selon la situation familiale dans le Plateau central. 
Figure 7 : Répartition de la fréquence d'utilisation des plantes médicinales selon l'habitat dans le Plateau central.

Fréquence d'utilisation des plantes médicinales : Les enquêtes auprès de la population de la dite région ont permis de recenser 51 espèces médicinales récoltées directement par l'utilisateur ou achetées auprès des herboristes, regroupées en 47 genres et appartenant à 32 familles. Les espèces les plus utilisées par la population locale sont: Opuntia ficus indica, Myrtus communis, Lavandula officinalis, Lavandula stoechas, Olea

europaea, Punica granatum et Eugenia cariophyllata. Le tableau 1 expose les plantes utilisées par la population pour traiter les affections dermatologiques. Les familles sont présentées par ordre alphabétique. Pour chaque plante recensée, nous donnons le nom scientifique, la famille, le nom commun, le nom arabe ou amazigh et la fréquence d'utilisation.

Tableau 1 : Liste des plantes médicinales, utilisées contre les affections dermatologiques, recensées dans le plateau central marocain.

\begin{tabular}{|c|c|c|c|c|}
\hline Famille & Nom scientifique & Nom commun & Nom arabe ou amazigh & Fréquence \\
\hline \multirow{2}{*}{ Amaryllidaceae } & Allium cepa & Oignon & Bassla & 11 \\
\hline & Allium sativum & Ail & Touma & 1 \\
\hline Anacardiaceae & Pistacia atlantica & Pistachia de l'atlas & Btam & 4 \\
\hline Apiaceae & Magydaris panacifolia & Magydaris & Frifra & 1 \\
\hline Aristolochiaceae & Aristolochia baetica & Aristoloche & Brezetam & 6 \\
\hline Asparagaceae & Urginea maritima & Basal eddib & Scille maritime & 1 \\
\hline \multirow[t]{5}{*}{ Asteraceae } & Calendula arvensis & Souci des champs & Jamera & 2 \\
\hline & Cynara cardunculus & Cardon & Kharechef & 1 \\
\hline & Cynara humilis & Cynara & Taymat & 6 \\
\hline & Echinops spinosus & Echinops & Taskra & 2 \\
\hline & Matricaria chamomilla & Camomille & Babounje & 3 \\
\hline \multirow[t]{2}{*}{ Brassicaceae } & Lepidium sativum & Creson alénois & Habe rechad & 5 \\
\hline & Moricandia arvensis & Chou des champs & Jrjir & 4 \\
\hline Cactaceae & Opuntia ficus indica & Figuier & Zeaboul & 41 \\
\hline Caryophyllaceae & Saponaria officinalis & Saponaire & Tighchet & 2 \\
\hline Cucurbitaceae & Ecballium elaterium & Concombre d'âne & Fgouss Ihmir & 7 \\
\hline Cyperaceae & Cyperus rotundus & Piri piri & Tarra & 8 \\
\hline Euphorbiaceae & Ricinus communis & Ricin & Krank & 2 \\
\hline \multirow[t]{2}{*}{ Fabaceae } & Astragalus gummifer & Epiaire & Ktira & 4 \\
\hline & Ceratonia siliqua & Caroubier & Slighwa & 2 \\
\hline Juncaceae & Juncus acutus & Jonc & Ssmar & 1 \\
\hline \multirow[t]{8}{*}{ Lamiaceae } & Ajuga iva & Ivette & Chendgûra & 6 \\
\hline & Lavandula officinalis & Lavande officinale & Khezama & 25 \\
\hline & Lavandula stoechas & Lavande stoechade & Helhal & 25 \\
\hline & Ocimum basilicum & Basilic & Hebak & 1 \\
\hline & Salvia verbenaca & $\begin{array}{l}\text { Sauge à feuilles de } \\
\text { verveine }\end{array}$ & Khayata & 5 \\
\hline & Thymus maroccanus & Thym & Zeitra & 2 \\
\hline & Thymus Zygis & Thym & Zeitra & 2 \\
\hline & Vitex agnus-castus & Gattilier & Kharwaâ & 5 \\
\hline \multirow[t]{2}{*}{ Lythraceae } & Lawsonia inermis & Henné & Henna & 8 \\
\hline & Punica granatum & Grenadier & Reman & 16 \\
\hline
\end{tabular}



traitement des affections dermatologiques dans le plateau central marocain

\begin{tabular}{l|l|l|l|l}
\hline \multirow{2}{*}{ Myrtaceae } & Eugenia cariophyllata & Girofle & Krenefel & 12 \\
\cline { 2 - 5 } & Myrtus communis & Myrthe & Rihan & 38 \\
\hline Nitrariaceae & Peganum harmala & Harmel & Harmel & 11 \\
\hline Oleaceae & Olea europaea & Oleastre & Zitoun & 18 \\
\hline Pinaceae & Pinus sylvestris & Pin & Tayda & 1 \\
\hline Plantaginaceae & Plantago major & Grand plantain & Messassa & 2 \\
\hline Poaceae & Avena sativa & Avoine cultivée & Khertal & 1 \\
\cline { 2 - 5 } & Phragmites communis & Kssab & Roseau & 6 \\
\hline Polygonaceae & Polygonum maritimum & Renouée maritime & Zyata & 6 \\
\hline Ranunculaceae & Nigella sativa & Nigelle & Sanouj & 2 \\
\hline Rhamnaceae & Zizyphus vulgaris & Zizyphus & Zefzouf & 1 \\
\hline Rosaceae & Rosa damascena & Rose de damas & Wared & 5 \\
\cline { 2 - 5 } & Rubus ulmifolius & Ronce & Aalik & 7 \\
\hline Salicaceae & Populus alba & Peuplier blanc & Safsaf & 2 \\
\hline Solanaceae & Mandragora automnalis & Mandragore & Bîid el-ghol & 10 \\
\hline Tamaricaceae & Tamarix aphylla & Tamaris & Takawet & 4 \\
\hline Thymelaeaceae & Daphne gnidium & Garou & Lzaze & 8 \\
\hline Verbenaceae & Lippia citriodora & Verveine & Louiza & 1 \\
\hline Zygophyllaceae & Zygophyllum gaetulum & Zygophylle & Äakaya & 2 \\
\hline & & & & \\
\hline
\end{tabular}

Analyse floristique : Les espèces végétales inventoriées sont réparties en quarante-sept genres et trente deux familles. Les familles ayant la plus grande diversité spécifique sont les Lamiacées (20,52\%) avec 71 espèces viennent ensuite des Myrtacées (14,45\%) avec 50 espèces, des Cactacées (11,84\%) avec 41 espèces, suivies des Lythracées avec $(6,93 \%)$ avec 24 espèces (figure 8).

\section{Fréquence}

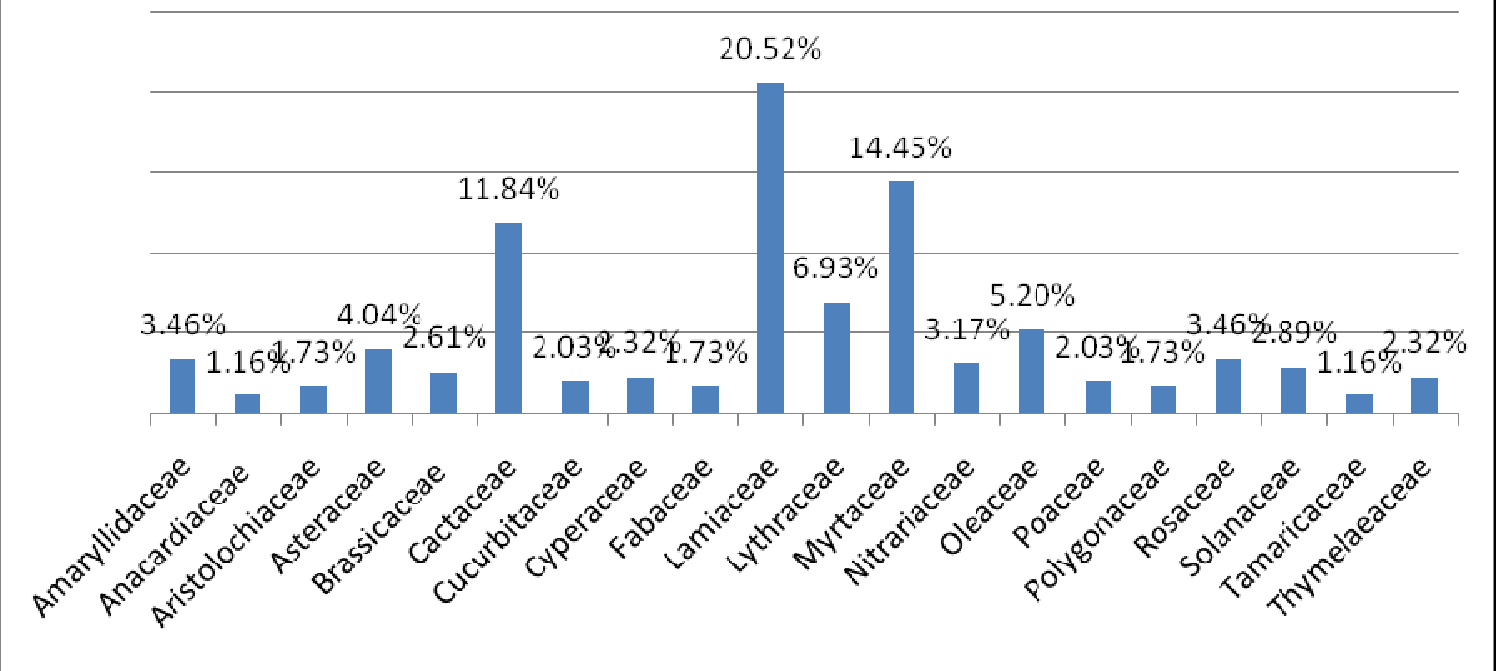

Figure 8 : les familles botaniques les plus utilisées dans le traitement des affections dermatologiques.

Utilisation thérapeutique des plantes médicinales

Selon la partie utilisée : Divers organes des plantes sont utilisés par la population pour la satisfaction de leurs besoins thérapeutiques, allant des feuilles, des tiges, des fruits, des racines, aux écorces, fleurs ou les inflorescences, et parfois même la plante entière. Dans la zone d'étude, les feuilles sont les organes les plus utilisés avec $(39,33 \%)$, suivies des tiges, racines, fruits, écorces, 
partie aérienne, graines, plantes entières, rhizomes,

fleurs, et des inflorescences avec un pourcentage de
$12,20 \%, 10,67 \%, 10,67 \%, 8,54 \%, 6,10 \%, 5,18 \%, 3,96 \%$, $2,44 \%, \quad 0,61 \%$,

$0,30 \%$

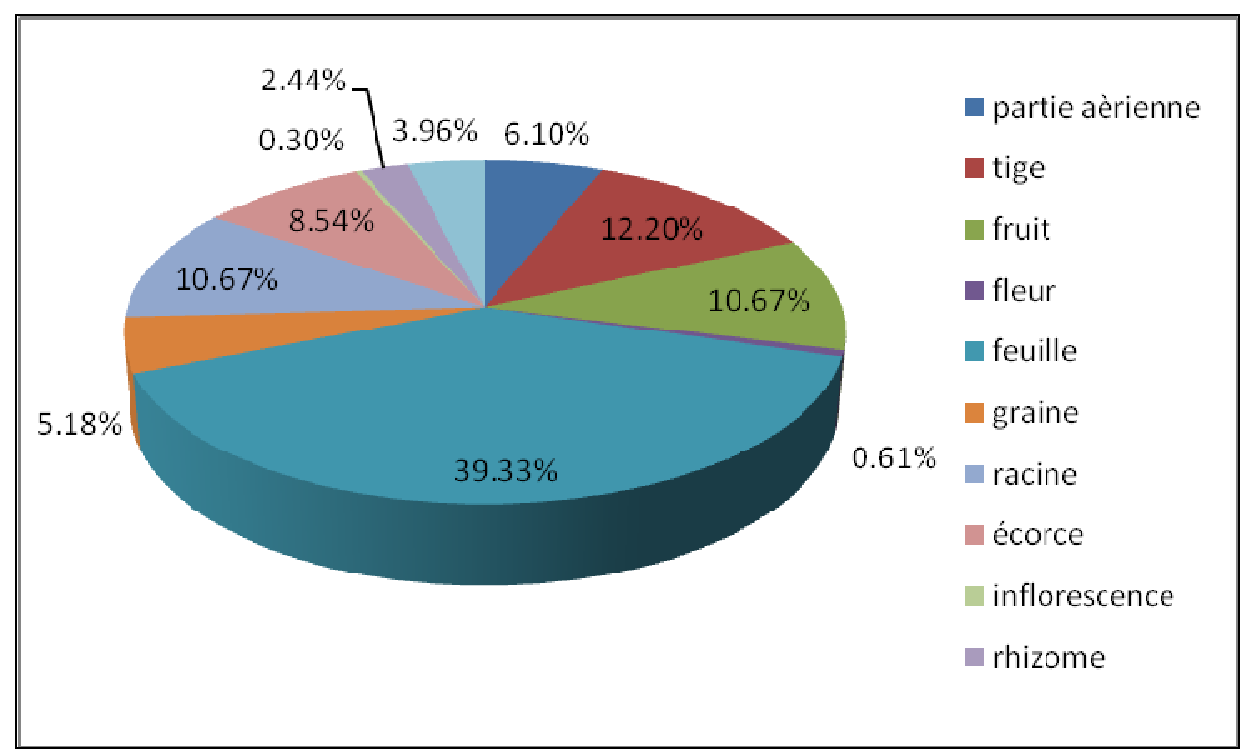

Figure 9 : Répartition des pourcentages de différentes parties utilisées des plantes médicinales contre les affections dermatologiques

Selon le mode de préparation : Différentes pratiques thérapeutiques sont employées par la population locale, pour le traitement des affections dermatologiques. La préparation en poudre est le mode le plus pratiqué avec un pourcentage de $58,66 \%$. Suivie du cataplasme, l'infusion et la décoction avec des pourcentages de $58,66 \%, 29,48 \%, 11,25 \%$ et $0,61 \%$.

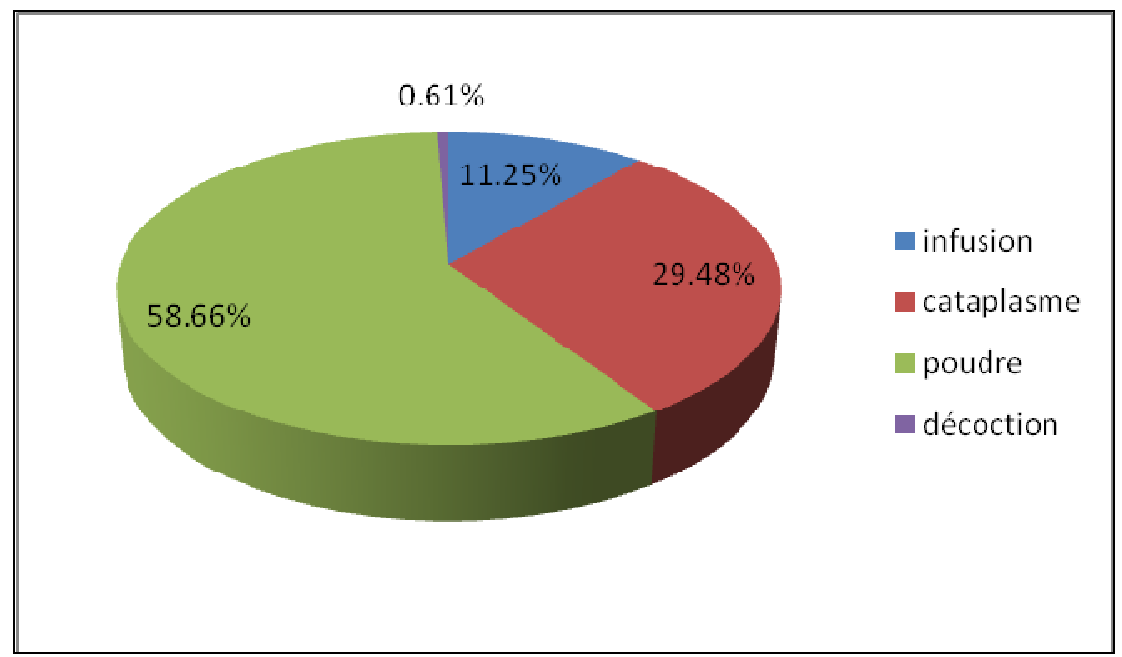

Figure 10 : Répartition des pourcentages des modes de préparation des plantes médicinales utilisées contre les affections dermatologiques

\section{CONCLUSION}

L'importance des plantes médicinales ne cesse d'augmenter en relation, d'une part, avec la forte

augmentation de la demande mondiale enregistrée ces dernières décennies pour les plantes médicinales et leurs 
produits dérivés et, d'autre part, avec le nombre croissant d'utilisateurs et la diversité des domaines de leur valorisation. L'étude ethnobotanique réalisée au plateau central marocain, nous a permis de mettre en évidence l'importante place de la phytothérapie traditionnelle. Les informations acquises, à partir des fiches questionnaires et l'analyse floristique menée sur le terrain, nous ont aidé à recenser 51 espèces végétales regroupées en 47 genres et appartenant à 32 familles dont les plus citées sont les Lamiacées, les Myrtacées, les Cactacées et les Lythracées. Les feuilles constituent la partie la plus utilisée avec un pourcentage de $(39,33 \%)$. La préparation en poudre est le mode le plus pratiqué avec un pourcentage de $(58,66 \%)$. Cette étude des flores médicinales vise la valorisation et la promotion de ce patrimoine culturel qui, finalement, permettra de valoriser la biodiversité et contribuer au développement et au bienêtre de la population locale.

\section{REMERCIEMENTS}

Nous tenons à remercier toute la population du plateau central, qui a bien voulu participer à notre enquête.

\section{RÉFÉRENCES BIBLIOGRAPHIQUES}

Adossides A., 2003. La filière Plantes Aromatiques \& Médicinales, FAO Projet; Assistance au Recensement Agricole. $70 \mathrm{p}$.

Beaudet G., 1969. Le plateau central marocain et ses bordures, étude géomorphologique. Thèse Lettres. Paris. 478p.

Combe M., Ferre M., Thauvi J., 1975. Ressource en eau du Maroc (tome 2), plaines et bassins du Maroc Atlantique (meseta central et meseta côtière), note de mémoire du service de géologie $\mathrm{N}^{\circ} 231$. p146- p17.

Fennane M., Ibn Tattou M., Mathez J., Ouyahya A., Eloualidi J., 1999. Flore pratique du Maroc, manuel de détermination des plantes vasculaire. Volume 1 : ptéridophyte - gymnospermes, angiosperme (Lauraceae-Neuradaceae). Travaux de l'institut scientifique, série botanique $n^{\circ}$ 36. Rabat. 558p.

Fennane M., Ibn Tatou M., 2005. Flore vasculaire du Maroc inventaire et chorologie (Volume1). Travaux De L'institut Scientifique, Série Botanique $\mathrm{N}^{\circ}$ 37. Rabat. 483p.

Fennane M., Ibn Tattou M., Ouyahya A., Eloualidi J., 2007. Flore pratique du Maroc, manuel de détermination des plantes vasculaire. Volume 2 : angiosperme (Leguminoseae- Lentibulariaceae). Travaux de l'institut scientifique, série botanique $n^{\circ}$ 38. Rabat. 636p.

Fennane. M., Ibn Tattou. M., Eloualidi. J., 2014. Flore pratique du Maroc, manuel de détermination des plantes vasculaires. Volume 3: Dicotylédones, Monocotylédones. Travaux de l'institut scientifique. Université Mohamed V. Rabat. 793p.

Quezel P., Santa S., 1962a. Nouvelle flore de l'Algérie et des Régions désertiques méridionales, (Tome I).
Edition centre national de la recherche scientifique. 565p.

Quezel P., Santa S., 1962b. Nouvelle flore de l'Algérie et des Régions désertiques méridionales, (Tome II). Edition centre national de la recherche scientifique. p571-p1165.

Selles Chaouki., 2012. Valorisation d'une plante médicinale à activité antidiabétique de la région de Tlemcen: Anacyclus pyrethrum L, Application de l'extrait aqueux à l'inhibition de corrosion d'un acier doux dans $\mathrm{H} 2 \mathrm{SO} 40.5 \mathrm{M}$. Thèse de pharmacie. 214p. 